\title{
No arc-connected treelike continuum is the 2-to-1 image of a continuum
}

\author{
by \\ Jo Heath (Auburn, AL) and Van C. Nall (Richmond, VA)
}

\begin{abstract}
In 1940, O. G. Harrold showed that no arc can be the exactly 2-to-1 continuous image of a metric continuum, and in 1947 W. H. Gottschalk showed that no dendrite is a 2-to- 1 image. In 2003 we show that no arc-connected treelike continuum is the 2-to-1 image of a continuum.
\end{abstract}

1. Introduction. Perhaps the biggest question in the category of 2-to-1 maps defined on metric continua is whether or not it is possible for a treelike continuum to be an image [9]. The spectrum of treelike continua goes from simple arcs to hereditarily indecomposable continua. Much is known at the ends of the spectrum, and little is known in the middle.

Perhaps the first result was Harrold's [4] 1940 result that arcs cannot be 2-to-1 images and this was generalized to dendrites (uniquely arc-connected Peano continua) by Gottschalk [3] in 1947. Much more recently, in 1987, Smithson [10] showed that if the domain continuum is arcwise connected, then it cannot map 2-to-1 onto an arc-connected treelike continuum (a dendroid). Last year the authors showed that if the domain continuum is hereditarily decomposable, then it cannot map 2-to-1 onto a dendroid [6]. As you can see by the title, we have removed the restriction of hereditary decomposability. (Although this current paper supercedes our previous paper, we use heavily the theorems and proofs from that paper.) Thus we now know that no arc-connected treelike continuum can be the 2-to- 1 image of a metric continuum.

What happens at the other end of the spectrum? If the treelike continuum is hereditarily indecomposable, it is known [5] that it cannot be a 2-to-1 image. If every proper subcontinuum of an indecomposable treelike

2000 Mathematics Subject Classification: Primary 54C10.

Key words and phrases: 2-to-1 map, dendroid, treelike, indecomposable continuum. 
continuum is an arc, then again [2] it cannot be a 2-to- 1 image; this class of continua includes all Knaster continua and the Ingram continua [7].

For the middle of the spectrum, it is known [2] that if a treelike continuum is the finite union of subcontinua each of which is not a 2-to-1 image, and no two of which intersect in more than one point, then the union also is not a 2-to-1 image. So, using this structure many hybrid treelike continua can be shown not to be 2-to-1 images.

It would seem, then, that the next logical step is to prove (or, if one must, disprove) either of the following conjectures:

Conjecture 1. No indecomposable treelike continuum can be the 2-to-1 image of a continuum.

ConjeCture 2. No hereditarily decomposable treelike continuum can be the 2-to-1 image of a continuum.

\section{Simple maps from indecomposable continua to dendroids.} We know [6] that if a continuum $X$ is hereditarily decomposable then there is no 2-to- 1 map from $X$ onto a dendroid. Therefore we naturally consider the case when $X$ contains indecomposable continua. A 2-to-1 map defined on $X$, when restricted to an indecomposable subcontinuum $I$, is a simple map (meaning each point inverse has at most 2 elements) from $I$ onto a dendroid. In this section we study these simple maps. There is an amazing amount of structure imposed on an indecomposable continuum if it admits a simple map onto a dendroid - perhaps contradictory structure.

Question 1 ([6]). Does there exist an indecomposable continuum that admits a simple map onto a dendroid?

In [8] Piotr Minc constructed a finite-to-one map from the Knaster buckethandle space onto a dendroid; in fact, each point inverse had at most 3 inverse points. But some points did have three inverse points. He also showed there is no simple map from an indecomposable continuum onto a planar dendroid, and that no Knaster type indecomposable continuum could be the domain of a simple map onto a dendroid. In the final section of this paper we generalize Minc's result to show that an indecomposable continuum with at least three arc-connected composants does not admit a simple map onto a dendroid.

In the same paper, Minc introduced the idea of a bottleneck continuum in a dendroid.

Definition 1 (Minc [8]). A subcontinuum $C$ of a dendroid $X$ is called a bottleneck continuum if there are two nonempty disjoint open sets $U$ and $V$ such that, for each $u \in U$ and $v \in V$, the arc $u v$ intersects $C$. 
TheOREM 1 (Minc [8]). Every nondegenerate dendroid $X$ contains points $a, b$ and $w \in a b \backslash\{a, b\}$ with the property that for every positive number $\varepsilon$ there is a continuum $C_{\varepsilon} \subset X$ and there are two open sets $U_{\varepsilon} \subset X$ and $V_{\varepsilon} \subset X$ such that

(1) $a \in U_{\varepsilon}$,

(2) $b \in V_{\varepsilon}$,

(3) $w \in C_{\varepsilon}$,

(4) $\operatorname{diam}\left(C_{\varepsilon}\right)<\varepsilon$, and

(5) $u v \cap C_{\varepsilon} \neq \emptyset$ for any $u \in U_{\varepsilon}$ and $v \in V_{\varepsilon}$.

In this paper we refer to the point $w$ in Theorem 1 as a center of $X$. If $a$ and $b$ are two points in a dendroid, then $a b$ will denote the unique arc in the dendroid with endpoints $a$ and $b$. If $f$ is a simple map defined on a continuum containing the point $p$, then $\widehat{p}$ denotes the twin point of $p$, i.e. the other point in the domain that maps the same as $p$. Of course, if $f$ is not 2-to-1 then some twins do not exist. Similarly, two disjoint sets in the domain of a simple map are twin sets if their images are the same. Other definitions may be found in the glossary at the end of the paper.

We often use the fact that dendroids are hereditarily unicoherent and arcwise connected and we use a result [1] of Borsuk that the closure of a nested union of arcs in a dendroid is also an arc. Thus if the collection of nested arcs all have a common endpoint then there is at most one new point in the closure of the union, the new endpoint.

Lemma 1. Let $f: I \rightarrow E$ be a simple map from an indecomposable continuum to a dendroid. If $K$ is a composant of $I$ then $f(K)$ is arcwise connected.

Proof. If $x$ and $y$ are points in $K$ then there is a proper subcontinuum $S$ of $I$ containing $x$ and $y$ that is contained in $K$. The image $f(S)$ is a subdendroid of $E$ containing $f(x)$ and $f(y)$ and hence contains an arc from $f(x)$ to $f(y)$.

Lemma 2. Let $f: I \rightarrow E$ be a simple map from an indecomposable continuum to a dendroid. Let $c$ be a center of $E$, and let $K$ be a composant of $I$. Then, for each $y \in f(K), c y \backslash\{c\} \subset f(K)$, and, if $K$ is disjoint from $f^{-1}(c)$, there is a $z \in c y \backslash\{c\}$ such that $f^{-1}(z) \subset K$, and $\left|f^{-1}(z)\right|=2$.

Proof. Using the construction of Minc for the center $c=f(p)$, there are points $a$ and $b$ in $E$, disjoint open sets $U_{n}$ and $V_{n}$ containing $a$ and $b$ and a continuum $C_{n}$ containing $f(p)$, for each positive integer $n$, that satisfy the construction for $e_{n}=1 / 2^{n}$. Without loss of generality, we assume that $1 / 2$ is less than the distance from $y=f(x)$ to $f(p)$. The open sets can be chosen to be nested, $U_{n+1} \subset U_{n}$ and $V_{n+1} \subset V_{n}$, as well as the continua $C_{n+1} \subset C_{n}$. For each positive integer $n$, a point $f\left(x_{n}\right)$ can be found in $C_{n} \cap f(K)$. To see 
this, note that the image of $K$ is dense in $E$ and thus intersects, for each $n$, both $U_{n}$ and $V_{n}$, and that from Lemma 1 we know there is an arc in $f(K)$ from $U_{n}$ to $V_{n}$. From the definition of bottleneck continuum, this arc intersects $C_{n}$. Now, since the continua $\left\{C_{n}\right\}$ are nested, we see that the point $f\left(x_{n+1}\right) \in C_{n+1}$ is also in $C_{n}$. Thus for each $n>1$, there is an arc $A_{n}$ in the dendroid $C_{n}$ from $f\left(x_{n}\right)$ to $f\left(x_{n+1}\right)$. By Lemma $1, f(K)$ contains $A_{n}$ for each $n$. Since the diameter of $A_{n}$ is less than $1 / 2^{n}$, the union of the arcs, together with the limit point $\{f(p)\}$, contains an arc $B$ from $f\left(x_{1}\right)$ to $f(p)$ such that $B \backslash\{f(p)\} \subset f(K)$. By Lemma 1 the arc from $f(x)$ to $B$ is a subset of $f(K)$, and this arc union $B$ contains the arc $A$ from $f(x)$ to $f(p)$. Since $A$ is contained in $f(K) \cup\{f(p)\}$, the first conclusion of this lemma is established.

Now suppose $K$ is a composant of $I$ whose image $f(K)$ does not contain $f(p)$. Let $R=A \backslash\{f(p)\}$ and suppose $f$ is 1-to-1 on the set $N=$ $K \cap f^{-1}(R)$. Note that $K$, and hence $N$, maps onto $R$. Then $N$ is homeomorphic to $[0,1)$ and its external limit points can only be $p$ or $\widehat{p}$, if $\widehat{p}$ exists. The closure of $N$ then is an arc in $I$ connecting $x$ in one composant to $p$ (or $\widehat{p}$ ) in another composant. This contradiction completes the proof.

Lemma 3. Suppose $f$ is a simple map from an indecomposable continuum $I$ to a dendroid $E$, and $f(p)$ is a center of $E$. If $K$ is a composant of $I$ containing neither $p$ nor $\widehat{p}$, then $f(K)$ is an arc component of $E \backslash\{f(p)\}$, and $K=f^{-1}(f(K))$.

Proof. It follows from Lemma 1 that $f(K)$ is arc-connected and, since $f(p)$ is not in $f(K)$, there is an arc component $\alpha$ of $E \backslash\{f(p)\}$ that contains $f(K)$. Now suppose another composant $K_{2}$ has a point $y$ that maps into $\alpha$. Choose any point $x$ in $K$. Since $\alpha$ is arc-connected, there is an $\operatorname{arc} B$ in $\alpha$ from $f(x)$ to $f(y)$. Let $C$ be the arc in $E$ from $B$ to $f(p)$. Since the arc in $E$ from $f(x)$ to $f(p)$ lies in $f(K)$ and the arc from $f(y)$ to $f(p)$ lies in $f\left(K_{2}\right)$, by Lemma 2, the arc $C$ is in $f(K) \cap f\left(K_{2}\right)$. But $C$ is an arc in $f(K)$ to the point $f(p)$ and $f$ cannot be 1 -to- 1 on the points of $K$ that map to $C$ (by Lemma 2, second part). This contradicts the fact that $f$ is simple.

COROllary 1. Suppose $f$ is a simple map from a continuum $X$ to a dendroid $E$ and $w b$ is an arc in $E$ such that for some indecomposable subcontinuum $J$ of $X, b$ is a center of $f(J)$. Then there is a composant $K$ of $J$ such that (1) $b \notin f(K)$, and (2) for every point $c$ in $f(K)$, the arc $b c$ intersects $w b$ only in the point $b$.

Proof. If the arc $w b$ intersects the dendroid $f(J)$ in a first point $a \neq b$, then $a b \backslash\{b\}$ lies in an arc component of $f(J) \backslash\{b\}$. From Lemma 3 we know that if $K$ is a composant of $J$ such that $f(K)$ does not contain $b$ (and there are uncountably many such composants) then $f(K)$ is one of the arc components of $f(J) \backslash\{b\}$ and we can simply choose one of these composants 
such that $f(K)$ does not contain $a b \backslash\{b\}$. If the arc $w b$ intersects the dendroid $f(J)$ in a first point that is $b$, then any composant of $J$ whose image misses $b$ will do.

Lemma 4. Suppose there is a simple map from an indecomposable continuum to a dendroid. Then the image dendroid has only one center.

Proof. Let $f$ be a simple map from the indecomposable continuum $I$ onto a dendroid $D$ and suppose $D$ has two centers $f(p)$ and $f(t)$, where $p$ and $t$ are not twins. Let $x$ be a point of $I$ in a composant $K$ that contains no point mapping to either $f(p)$ or $f(t)$. By Lemma 2, the arc $A$ from $f(x)$ to $f(t)$ cannot contain $f(p)$ since the arc lies in $f(K) \cup\{f(t)\}$, and the arc $B$ from $f(x)$ to $f(p)$ cannot contain $f(t)$. Hence, if $C$ denotes the interior of the arc in $E$ from $f(p)$ to $f(t)$, then $C \subset A \cup B$. Thus, by Lemma 2 again, $C$ is in the image of $K$. But there are uncountably many composants that could have been chosen instead of $K$ and there is only one arc in $E$ from $f(p)$ to $f(t)$.

Lemma 5. Suppose $f$ is a simple map from an indecomposable continuum I to a dendroid $E$, and $f(p)$ is the center of $E$. If I is irreducible about a pair of twin points that do not map to $f(p)$, then $p$ has a twin $\widehat{p}$ in $I$ and there are disjoint twin arcs in different composants of I from one of the twin points to $p$ and from the other to $\widehat{p}$.

Proof. Since $I$ is irreducible about the twin pair of points which we will name $x$ and $\widehat{x}$, they cannot be in the same composant. So if $K_{1}$ and $K_{2}$ are the composants containing $x$ and $\widehat{x}$ respectively, then $K_{1}$ and $K_{2}$ must both contain a point that maps to $f(p)$ by Lemma 3 , since their images are not disjoint. Suppose $p \in K_{1}$ and $\widehat{p} \in K_{2}$. It follows from Lemma 1 that both $K_{1}$ and $K_{2}$ map onto the arc $A$ in $E$ from $f(x)$ to $f(p)$. Thus each of $K_{1}$ and $K_{2}$ have, for each point $t \in A$, exactly one point that maps to $t$. This means that $f^{-1}(A) \cap K_{1}$ and $f^{-1}(A) \cap K_{2}$ are disjoint twin arcs as needed.

Lemma 6. If $f$ is a simple map from a continuum $X$ onto a dendroid and $f(x)=f(y)$, then either

(1) there is an arc in $X$ from $x$ to $y$, or

(2) there are disjoint twin arcs $x x^{\prime}$ and $y y^{\prime}$ in $X$ such that for some indecomposable continuum I in $X$ containing $x^{\prime}$ and $y^{\prime}, f\left(x^{\prime}\right)$ is the center of $f(I)$, or

(3) there is an indecomposable continuum $I$ in $X$ containing $x$ and $y$ such that $f(x)$ is the center of $f(I)$.

Proof. In [6] we show that if $f$ is a simple map from a hereditarily decomposable continuum $X$ onto a dendroid, and $f(x)=f(y)$, then there is an arc in $X$ connecting $x$ and $y$. 
The proof of this theorem implies that if $f$ is a simple map from a hereditarily decomposable continuum $X$ onto a dendroid, if $f(x)=f(y)$, and if $L$ is a continuum in $X$ irreducible about $x$ and $y$, then (1) $L=H \cup N$, two proper subcontinua of $L$ with $x \in H \backslash N$ and $y \in N \backslash H$, and (2) there are disjoint twin half-open arcs in $L, x x^{\prime} \backslash\left\{x^{\prime}\right\}$ and $y y^{\prime} \backslash\left\{y^{\prime}\right\}$, such that (i) $x x^{\prime} \backslash\left\{x^{\prime}\right\} \subset H \backslash N$, and (ii) $\left\{x^{\prime}\right\} \cup y y^{\prime} \subset N$, or the other way around. Then, if the new endpoints $x^{\prime}$ and $y^{\prime}$ are not equal (else there is an arc $f$ from $x$ to $y$ ), the construction continues in a subcontinuum of $N$ irreducible about $x^{\prime}$ and $y^{\prime}$. Furthermore, at each limit case, after infinitely many extensions, there continues to be an extension. The point is that so long as the irreducible continuum containing the new endpoints is decomposable, the process continues. More details can be found in Lemma 7 in $[6]$.

Therefore, if $f$ is a simple map from a continuum $X$ onto a dendroid, if $f(x)=f(y)$, and if there is no arc in $X$ connecting $x$ and $y$, then there are disjoint twin $\operatorname{arcs} x x^{\prime}$ and $y y^{\prime}$ in $X$ and an indecomposable subcontinuum $I$ in $X$ that is irreducible about $x^{\prime}$ and $y^{\prime}$. Then Lemma 5 implies that either $f\left(x^{\prime}\right)$ is the center for $f(I)$, or there are disjoint twin $\operatorname{arcs} x^{\prime} p$ and $y^{\prime} \widehat{p}$ in different composants of $I$ such that $f(p)$ is the center for $f(I)$. Contained in the disjoint twin sets $x x^{\prime} \cup x^{\prime} p$ and $y y^{\prime} \cup y^{\prime} \widehat{p}$ are disjoint twin $\operatorname{arcs} x p$ and $y \widehat{p}$.

Or, much easier, the degenerate arcs case occurs. This is the case where the disjoint twin arcs are just points because the original two points $x$ and $y$ are already in an indecomposable continuum $I$ and $f(x)$ is the center for $f(I)$.

Definition. Suppose $f: X \rightarrow D$ is a simple map from a continuum $X$ onto a dendroid $D$ and $a w$ is an $\operatorname{arc}$ in $D$. Then the endpoint $w$ will be called a fold point in aw if there is a sequence $w_{1}, w_{2}, \ldots$ in $a w \backslash\{w\}$ converging to $w$, there are two points in the preimage of $w_{i}$ for each $i$, and there is one point $x$ in the preimge of $w$ such that the sequence $\left\{f^{-1}\left(w_{i}\right)\right\}$ converges to $x$. Note that since the sequence $\left\{f^{-1}\left(w_{i}\right)\right\}$ can only have inverse points of $w$ as limit points, if $w$ happens to have only one inverse point, then $w$ will be a fold point by default.

Smithson [10] showed that if $f$ is a simple map defined on an $\operatorname{arc} A$ and $f(A)$ is uniquely arc-connected and such that the endpoints of $A$, denoted $e$ and $e^{\prime}$, are mapped to the same point, then the structure of the map is straightforward: there is a point $c$ in $A$ such that the image of $f$ is an arc with endpoints $f(e)$ and $f(c)$, and $f$ restricted to each of the two parts of $A$, $e c$ and $c e^{\prime}$, is a homeomorphism onto the image arc. Notice that this makes $f(c)$ a fold point in the arc from $f(e)$ to $f(c)$.

Lemma 7. Suppose $f: I \rightarrow E$ is a simple map from an indecomposable continuum $I$ onto a dendroid $E, w=f(p)$ is the center of $f(I)$, and $K$ is 
a composant of $I$ such that $f(K)$ does not contain $w$. Then there is a point $w^{\prime} \in f(K)$ such that $w^{\prime}$ is a fold point in $w w^{\prime}$.

Proof. Assume there is no $w^{\prime} \in f(K)$ such that $w^{\prime}$ is a fold point in $w w^{\prime}$. Let $\mathcal{T}$ be the set of all triples $T(b)=\left(b, S_{b}, F_{b}\right)$ where

(a) $b \in f(K)$,

(b) $S_{b} \subset w b \backslash\{b\}$ in $E$ with $b=\sup \left(S_{b}\right)$, and

(c) $F_{b}=\left\{I_{c} \mid c \in S_{b}\right\}$ is a collection of indecomposable continua in $K$ such that for each $c \in S_{b}$,

(i) $c$ is the center of $f\left(I_{c}\right)$,

(ii) $\left|f^{-1}(c) \cap I_{c}\right|=2$, and

(iii) if $c, c^{\prime} \in S_{b}$ and $c<c^{\prime}$, then there is a composant $K_{c}$ of $I_{c}$ such that $f\left(K_{c}\right)$ contains $c c^{\prime} \backslash\{c\}$ but does not contain $c$, and $I_{c^{\prime}} \subset K_{c}$.

Define an order on $\mathcal{T}$ by $T(b)<T(c)$ iff $w b \subset w c, S_{b} \subset S_{c}$, and if $I_{s} \in F_{b}$ and $I_{s^{\prime}}^{\prime} \in F_{c}$ with $s=s^{\prime}$, then $I_{s}=I_{s^{\prime}}^{\prime}$.

Observation 1. These definitions imply that if $T(b)<T(c)$ then there is no arc in $I_{b}$ whose image contains both $b$ and $c$ since this arc would connect points from two composants in $I_{b}$.

First we need to see that $\mathcal{T}$ is not empty. Notice that if there exists a twin pair $\left\{b_{1}, b_{2}\right\}$ of points in $K$ that is not contained in an arc in $K$, then for any continuum $L$ in $K$ that contains $b_{1}$ and $b_{2}$, according to Lemma 6 , there is an indecomposable continuum $I_{w_{1}}$ in $L$, where $w_{1}$ denotes the center of $f\left(I_{w_{1}}\right)$, such that $\left|f^{-1}\left(w_{1}\right) \cap I_{w_{1}}\right|=2$. Let $K_{1}$ be any composant of $I_{w_{1}}$ that satisfies the conclusion of Corollary 1, i.e. $w_{1} \notin f\left(K_{1}\right)$ and if $w_{2}$ is any point in $f\left(K_{1}\right)$ then $w w_{1} \cap w_{1} w_{2}=\left\{w_{1}\right\}$

If $K_{1}$ contains a twin pair that is not contained in an arc in $K_{1}$, the process of constructing the nested arcs continues. If the process continues for infinitely many steps the result will be a nested sequence of arcs $\left\{w w_{i}\right\}$ whose closure is an arc $w b$ such that the triple $T(b)=\left(b, S_{b}, F_{b}\right)$, where $S_{b}=\left\{w_{1}, w_{2}, \ldots\right\}$ and $F_{b}=\left\{I_{w_{1}}, I_{w_{2}}, \ldots\right\}$, satisfies all of the conditions above. That is, if this process continues, then the collection $\mathcal{T}$ is not empty.

We will show that this process continues. For assume it is not true. That is, assume that for some $i$ each twin pair in the composant $K_{i}$ of the indecomposable continuum $I_{c}$ is contained in an arc that is contained in $K_{i}$. Let $Q=\left\{b \in f\left(K_{i}\right):\left|f^{-1}(b) \cap K_{i}\right|=2\right\}$. It follows from Lemma 2 that $Q$ is not empty. For each $b \in Q$ let $A_{b}$ be the irreducible arc in $K_{i}$ containing $f^{-1}(b)$ and let $w_{b}$ be the element of $f\left(K_{i}\right)$ such that $f\left(A_{b}\right)=b w_{b}$. Note that $w_{b}$ is a fold point of $b w_{b}$, and if $w_{b}$ is not in $w b$, then $w_{b}$ is a fold point of $w w_{b}$, contrary to our assumption. So assume that $w_{b} \in w b$ for each $b \in Q$. It follows that $f\left(A_{b}\right) \subset w b$ for each $b \in Q$. 
Now we shall show that this leads to a contradiction. First we prove that

(*) if $M$ is a continuum in $f\left(I_{c}\right)$ containing $c$ and the point $y$ in $f\left(K_{i}\right)$, then there is a continuum in $f^{-1}\left(M \cap f\left(K_{i}\right)\right)$ that contains $f^{-1}(y)$.

We may assume that $f^{-1}(y)$ is not connected and both of its points are in $K_{i}$ since $f^{-1}\left(f\left(K_{i}\right)\right)=K_{i}$. Thus it is enough to show that $A_{y}$ is a continuum with the desired properties. By our assumption $f^{-1}(y) \in A_{y}$. Above it was noted that $f\left(A_{y}\right) \subset y c \backslash\{c\}$. But $y c \subset M$ and $y c \backslash\{c\} \subset f\left(K_{i}\right)$, hence $y c \backslash\{c\} \subset M \cap f\left(K_{i}\right)$. Therefore $A_{y} \subset f^{-1}\left(M \cap f\left(K_{i}\right)\right)$, which ends the proof of $(*)$.

The dendroid $f\left(I_{c}\right)$ is decomposable, so there are proper subcontinua $H$ and $L$ whose union is $f\left(I_{c}\right)$. If one of them, say $H$, does not contain $c$, let $H^{\prime}=H \cup x c$ where $x$ is a point of $H$. Then $H^{\prime}$ is still proper because $H^{\prime}$ is locally connected at points of $x c$ near $c$ and the whole space $f\left(I_{c}\right)$ is not locally connected at any point different from $c$. This is because there are uncountably many arc components of $f\left(I_{c}\right) \backslash\{c\}$ that are dense in $f\left(I_{c}\right)$. Therefore $f\left(I_{c}\right)=M_{0} \cup M_{1}$, where each $M_{j}, j=0,1$, is a proper subcontinuum of $f\left(I_{c}\right)$ containing $c$.

In a dendroid the intersection $M_{j} \cap f\left(K_{i}\right)$ of two arcwise connected sets is arcwise connected, hence connected. The mapping $f: f^{-1}\left(M_{j} \cap f\left(K_{i}\right)\right) \rightarrow$ $M_{j} \cap f\left(K_{i}\right)$ is quotient (i.e. a set is open in the image iff its preimage is open in the domain). This follows from the straightforward lemma that if $f$ is a map from a compact space $I$ onto $E$ and $L$ is a subset of $E$, then the restricted map $f: f^{-1}(L) \rightarrow L$ is quotient. Since $f: f^{-1}\left(M_{j} \cap f\left(K_{i}\right)\right) \rightarrow$ $M_{j} \cap f\left(K_{i}\right)$ is quotient it satisfies the property of all quotient maps that if the image $C$ is connected and for each $y \in C$ there is a connected subset of the domain containing $f^{-1}(y)$, then the domain is connected. Thus each set $f^{-1}\left(M_{j} \cap f\left(K_{i}\right)\right)$ is connected. Since $K_{i} \subset I_{c}$ we have $f\left(K_{i}\right)=\left(M_{0} \cap\right.$ $\left.f\left(K_{i}\right)\right) \cup\left(M_{1} \cap f\left(K_{i}\right)\right)$. As $M_{j}$ is a proper subcontinuum of $f\left(I_{c}\right)$ we infer that $\operatorname{cl}\left(f^{-1}\left(M_{j} \cap f(K i)\right)\right)$ is a proper subcontinuum of $I_{c}$. It follows that $I_{c}=\operatorname{cl}\left(K_{i}\right)=\operatorname{cl}\left(f^{-1}\left(M_{0} \cap f\left(K_{i}\right)\right)\right) \cup \operatorname{cl}\left(f^{-1}\left(M_{1} \cap f\left(K_{i}\right)\right)\right)$ contrary to the indecomposability of $I_{c}$.

So $\mathcal{T}$ is not empty. If $T\left(b_{\lambda}\right)_{\lambda \in \Lambda}$ is a linearly ordered subcollection of $\mathcal{T}$ and

$$
w b=\overline{\bigcup_{\lambda \in \Lambda} w b_{\lambda}}, \quad S_{b}=\bigcup_{\lambda \in \Lambda} S_{b_{\lambda}}, \quad F_{b}=\bigcup_{\lambda \in \Lambda} F_{b_{\lambda}},
$$

then $T(b)=\left(b, S_{b}, F_{b}\right) \in \mathcal{T}$, and $T\left(b_{\lambda}\right)<T(b)$ for each $\lambda \in \Lambda$.

It follows from Zorn's lemma that there is a maximal triple $T(m)$. Let $M=\bigcap_{c \in S_{m}} F_{c}$. Suppose $\left|f^{-1}(m) \cap M\right|=1$. We know that $\left|f^{-1}(c) \cap I_{c}\right|=2$ for each $c \in S_{m}$ and that $m=\sup \left(S_{m}\right)$. Therefore, $m$ is a fold point in $w m$, and we have assumed there are none. So $\left|f^{-1}(m) \cap M\right|=2$. 
We will extend the maximal triple $T(m)$ to complete the contradiction. Using $M$ as the continuum $X$ in Lemma 6 , and using $x$ and $y$ to denote the two inverse points of $m$, there are only three possibilities: (1) there is an arc in $M$ from $x$ to $y,(2)$ there are disjoint twin $\operatorname{arcs} x x^{\prime}$ and $y y^{\prime}$ in $M$ and an indecomposable continuum $J$ in $M$ containing $x^{\prime}$ and $y^{\prime}$ such that $f\left(x^{\prime}\right)$ is the center of $f(J)$, or (3) there is an indecomposable continuum $J$ in $M$ containing $x$ and $y$ such that $f(x)$ is the center of $f(J)$.

First we will show that case (1) cannot hold. On the contrary, suppose $A_{m}$ denotes an arc in $M$ from $x$ to $y$ and let $m z$ be the image of $A_{m}$. We have seen that unless $m z$ lies in $w m, z$ will be a fold point of $w z$. So $m z$ is contained in $w m$, and there is a point $c$ in $m z$ such that the inverse of $m c$ is the union of two disjoint twin $\operatorname{arcs} A$ and $B$ in $M$. But $m c \subset w m$ means that there are two elements of $S_{m}$, say $a$ and $b$ with $T(a)<T(b)$, that lie in $m c$. Since $A$ is a subset of $M$, it is a subset of $I_{a}$; but the $\operatorname{arc} A$ contradicts Observation 1.

If (2) occurs, the same argument implies that the image of the disjoint twin $\operatorname{arcs}$ in $M$ cannot backtrack, i.e. the new arc $m f\left(x^{\prime}\right)$ intersects $w m$ only in the point $m$.

So, whether case (2) holds or case (3) holds, we have an arc $w f\left(x^{\prime}\right)$ that contains $w m$ and an indecomposable continuum $J$ in $M$, containing $x^{\prime}$ and $y^{\prime}$, such that $f\left(x^{\prime}\right)$ is the center of $f(J)$. (Note that in case (3), $x^{\prime}=x$ and so $f\left(x^{\prime}\right)=m$ ). Now, using the same construction in $J$ as was used at the beginning of the proof in $I$, and using Corollary 1 to choose a suitable composant of $J$ to work in, we have an element of $\mathcal{T}$ that properly contains $T(m)$. This contradiction completes the proof.

\section{Main result}

TheOrem 2. No dendroid is the image of a 2-to-1 map defined on a continuum.

Proof. On the contrary, suppose there is a 2-to- 1 map $f$ from a continuum $X$ onto a dendroid $D$. Let $a$ denote any point of $D$. We consider the collection $\mathcal{B}$ of all arcs $a w$ in $D$ where $w$ is a fold point in aw. Our plan is to show, using Zorn's lemma, that $\mathcal{B}$ must have a maximal element (with respect to the natural ordering of inclusion), and yet we can extend any element of $\mathcal{B}$ to a longer arc in $\mathcal{B}$. This contradiction will complete our proof.

First, we will show that $\mathcal{B}$ is non-empty, and at the same time give a preview of the constructions to come. We know from Lemma 6 that if $a$ is a point of $D$ and its inverse points are $a_{1}$ and $a_{2}$, then one of three things can happen. Either (1) there is an arc in $X$ with endpoints $a_{1}$ and $a_{2}$, or (2) there are disjoint twin $\operatorname{arcs} a_{1} w_{1}$ and $a_{2} w_{2}$ and an indecomposable 
continuum $I$ containing $w_{1}$ and $w_{2}$ such that $f\left(w_{1}\right)$ is the center of $f(I)$, or (3) (the degenerate arcs case (2)) there is an indecomposable continuum $I$ containing $a_{1}$ and $a_{2}$ such that $f\left(a_{1}\right)$ is the center of $f(I)$.

In case (1), we know by Smithson's construction that there is a point $c$ in $A$ such that $f(c)$ is a fold point in the arc af $(c)$; so af $f(c)$ belongs to $\mathcal{B}$.

For case (2) (case (3) is similar), we use Lemma 7 with the simple map $f$ restricted to $I$. For every composant $K$ of $I$ whose image misses $w$ there is a point $w^{\prime} \in f(K)$ such that $w^{\prime}$ is a fold point in the arc $w w^{\prime}$ in $f(I)$. From Corollary 1 , there a composant $K$ if $I$ such that $w \notin f(K)$ and the arc $w w^{\prime}$ intersects $a w$ only in the point $w$. Hence, $w^{\prime}$ is a fold point of the arc $a w^{\prime}$ in $\mathcal{B}$. Thus, in all three cases, we have shown that $\mathcal{B}$ is not empty.

If $\left\{a t_{\lambda}\right\}_{\lambda \in \Lambda}$ is a linearly ordered subcollection of $\mathcal{B}$, then the closure of the union of this subcollection is an arc [1], say $a w^{\prime}$. The arc $a w^{\prime}$ is in $\mathcal{B}$ because the new endpoint $w^{\prime}$ is a limit point of a sequence of fold points and it is easy to see that such a limit of fold points is a fold point.

So, there is a point $w$ such that $a w$ is a maximal element in $\mathcal{B}$. Then, from the definition of fold point, there is a sequence $\left\{t_{i}\right\}$ of points on the arc aw converging to $w$ and one inverse point of $w$, say $w_{2}$, that is not a limit point of $\bigcup\left\{f^{-1}\left(t_{i}\right)\right\}$. Notice that the definition of fold point implies that for any point $y$ in $a w$, the inverse of the arc $y w$ cannot be the disjoint union of twin arcs.

From Lemmas 6 and 7, there are three ways, depending on the 3 cases described earlier, to construct an arc $w w^{\prime}$ such that $w^{\prime}$ is a fold point in $w w^{\prime}$. Our primary concern now will be that $a w$ and $w w^{\prime}$ intersect only in their common endpoint $w$, so that aw will be a proper subset of $a w^{\prime}$. In cases (1) and (2), the constructed arc $w w^{\prime}$ has an initial segment $w z$ whose inverse consists of two disjoint twin $\operatorname{arcs}$ in $X$. But from the definition of the fold point $w$ in $a w$, no final segment $y w$ in aw has an inverse consisting of disjoint twin arcs. We also know, since $D$ is a dendroid, that if $a w$ intersects $w w^{\prime}$ in more than their common endpoint then they intersect in some final segment $y w$ of $a w$. Hence in cases (1) and (2), the arc $a w^{\prime}$ in $\mathcal{B}$ properly contains the arc aw. In the third and last case, the degenerate arc case, there is, as in the construction to show $\mathcal{B}$ is nonempty, a composant of $I$ whose image contains a suitable arc $w w^{\prime} \backslash\{w\}$ where $w^{\prime}$ is a fold point of $w w^{\prime}$, and the new arc $w w^{\prime}$ intersects aw only in the point $w$. Hence, in all three cases, $w$ cannot be a fold point since aw is maximal.

4. Simple maps onto dendroids. In this final section we use facts established in the previous lemmas to say more about the structure of a simple map from an indecomposable continuum onto a dendroid, if, in fact, there is such a map. 
LEMMA 8. If two points of an indecomposable continuum are identified, the resulting continuum is still indecomposable.

Proof. Suppose $i$ denotes the map from the indecomposable continuum $I$ onto a continuum $I^{\prime}$ that identifies the points $p$ and $q$ of $I$. Let $x$ and $y$ denote two points from different composants of $I$ that are both also not in either the composant of $I$ containing $p$ or the one containing $q$. To see that $I^{\prime}$ has uncountably many composants and hence is indecomposable, we need to show that $i(x)$ and $i(y)$ remain in different composants of $I^{\prime}$. So suppose that there is a proper subcontinuum $C$ in $I^{\prime}$ containing both $i(x)$ and $i(y)$. If $C$ does not contain $i(p)$ then $i^{-1}(C)$ is homeomorphic to $C$ and so is a proper subcontinuum of $I$ containing $x$ and $y$. Hence $C$ contains $i(p)$. Consider the component $T$ of $C \backslash\{i(p)\}$ that contains $x$. Then $T$ goes to the boundary so $\{i(p)\}$ is in the closure of $T$ and this implies that either $p$ or $q$ is in the closure of $i^{-1}(T)$. But $i^{-1}(T)$ is connected (remember $i$ is 1-to- 1 on $i^{-1}(T)$ ) and its closure is a continuum containing both $x$ and either $p$ or $q$. But its closure is a proper subcontinuum of $I$ since $C$ is a proper continuum in $I^{\prime}$ that contains $i(p)$. This contradiction to the fact that $x$ and either $p$ or $q$ are in different composants of $I$ completes the proof.

THEOREM 3. Suppose $f$ is a map from an indecomposable continuum I onto a dendroid $D, f(p)$ is a center in $D$, and there is a composant $K$ of $I$ whose image does not contain $f(p)$ such that each pair of twin points in $K$ is contained in an arc in $K$. Then the map $f$ cannot be simple.

Proof. Suppose on the contrary that $I, D, f$, and $K$ exist satisfying the hypothesis, but $f$ is simple. First, we will consolidate the domain without changing the image by identifying in $I$ the two points $p$ and $\widehat{p}$, if indeed $\widehat{p}$ exists. Then the natural map based on $f$ defined on the new indecomposable continuum is a simple map onto the same dendroid and the set $K$ is still a composant whose image does not contain $f(p)$. In other words, without loss of generality we may assume that $\widehat{p}$ does not exist.

Let $A$ be an arc in $f(K) \cup\{f(p)\}$ with one endpoint $f(p)$. (See Lemma 2.) There are points $a$ and $b$ in $D$ such that these two points satisfy the conclusion of Theorem 1 for the center $f(p)$. From Theorem 1 again, $f(p)$ is on the arc $a b$ in $D$, and it is possible that either $a$ or $b$ is on the arc $A$. If so, choose a shorter $A$ so that neither $a$ nor $b$ is on $A$. We will need this fact later.

From Lemma 2 we know some points of $A$ have two point inverses; let $B=\left\{f(x) \in A|| f^{-1}(f(x)) \mid=2\right\}$. Note that $f(p)$ is not in $B$ since $p$ has no twin. From the hypothesis we know that there is an arc $x \widehat{x}$ in $K$ for each $f(x) \in B$. Let $\mathcal{A}$ denote this collection of arcs. We need to know now that $C=f^{-1}(A) \cup(\cup \mathcal{A})$ is connected. Suppose on the contrary that $C=H \mid L$, the disjoint union of separated sets. Note that no point $c$ of $A$ 
belongs to both $f(H)$ and $f(L)$ since that would mean that $c$ is in $B$ and the arc in $K$ between its inverse points would be in $C$ and intersect both $H$ and $L$. Hence the $\operatorname{arc} A$ is the disjoint union of $f(H) \cap A$ and $f(L) \cap A$. Since $A$ is connected, there is a convergent sequence of points $c_{i}$ of, say, $f(H) \cap A$ that converges to the point $c$ of $f(L) \cap A$. A convergent sequence of points in $H$ can be extracted from the sequence of point sets $f^{-1}\left(c_{i}\right)$, and this sequence must converge to an inverse of $c$ that is not in $L$. So $c$ is in $B$ and the arc in $C$ between its inverse points intersects both $H$ and $L$. This is a contradiction.

We now know that $\bar{C}$ is a continuum containing $p$ and many points of $K$. Hence $\bar{C}=I$, and since $f^{-1}(A)$ is compact and its complement dense, the union of the $\operatorname{arcs}$ in $\mathcal{A}$ is dense in $I$.

We now want to find a convergent subsequence of $\operatorname{arcs}$ in $\mathcal{A}$ that is still dense in $I$. Choose a point $q$ in a composant of $I$ other than $K$ and other than the composant containing $p$, and choose a convergent sequence of $\operatorname{arcs} S$ from $\mathcal{A}$ that has $q$ in its limiting set. Each of these arcs has twin endpoints that map to a point of $A$, and so the images of the $\operatorname{arcs}$ in $S$ are $\operatorname{arcs}$ in $D$ whose endpoints on $A$ converge to a point of $A$ and these arcs have the point $f(q)$ in their limiting set. Hence the limiting set of the image arcs must contain $f(p)$ since $f(q)$ is not in the same arc component of $D \backslash\{f(p)\}$ as the points of $f(K)$ (see Lemma 3). This means that the limiting set of the arcs in $S$ contains $p$, and since it is a continuum containing $p$ and $q$, it is the continuum $I$. Thus we have extracted from $\mathcal{A}$ a convergent sequence $S$ of arcs whose union is dense in $I$. If $S^{\prime}$ denotes the convergent sequence of image $\operatorname{arcs}\{f(x \widehat{x}) \mid x \widehat{x} \in S\}$ in $D$, the union of the elements of $S^{\prime}$ is dense in $D$.

For the rest of the proof we use the technique of Piotr Minc in his proof that no Knaster type indecomposable continuum admits a simple map onto a dendroid. Referring again to the bottleneck structure in $D$ set up in Theorem 1 for the center $f(p)$, we have points $a$ and $b$ with the property that for every positive number $\varepsilon$ there is a continuum $C_{\varepsilon} \subset D$ and there are two open sets $U_{\varepsilon} \subset D$ and $V_{\varepsilon} \subset D$ such that

(1) $a \in U_{\varepsilon}$,

(2) $b \in V_{\varepsilon}$,

(3) $f(p) \in C_{\varepsilon}$,

(4) $\operatorname{diam}\left(C_{\varepsilon}\right)<\varepsilon$, and

(5) $u v \cap C_{\varepsilon} \neq \emptyset$ for any $u \in U_{\varepsilon}$ and $v \in V_{\varepsilon}$.

Choose $\varepsilon$ small enough and open sets $U_{\varepsilon}$ and $V_{\varepsilon}$ small enough about $a$ and $b$ that the closures of all three are pairwise disjoint and the closures of both $U_{\varepsilon}$ and $V_{\varepsilon}$ miss $A$. (Recall that neither $a$ nor $b$ is in the arc $A$.) The sequence $S^{\prime}$ of $\operatorname{arcs}$ in $D$ is dense in $D$ and so there is a subsequence that has 
$a$ in its limiting set and a further subsequence that also has $b$ in its limiting set. Thus there is an arc $G$ in $S^{\prime}$ that has its initial point in $A$, and two other points, one in $U_{\varepsilon}$, and one in $V_{\varepsilon}$; but between these two points, by Theorem 1, there is a point of $C_{\varepsilon}$ in $G$. If $M$ denotes the continuum $C_{\varepsilon} \cup A$, then the arc $G$ starts in $M$, goes over to either $U_{\varepsilon}$ or $V_{\varepsilon}$, and then returns to $M$. But in a dendroid, the intersection of two continua such as $G$ and $M$ must be connected. This contradiction completes the proof.

The following is a consequence of Lemma 3 and Theorem 3.

COROLlary 2. If three composants of an indecomposable continuum I are arc-connected, then there is no simple map from I onto a dendroid.

5. Glossary. 1. A topological space is a continuum if it is compact, connected and metrizable.

2. A 2-to-1 map is crisp if the preimage of each proper subcontinuum in the image consists of two components, each of which maps homeomorphically onto the subcontinuum.

3. If $M$ is a continuum, a subcontinuum $N$ of $M$ is a $c$-set in $M$ provided $N$ is a subset of any subcontinuum of $M$ that contains both a point in $N$ and a point not in $N$.

4. A continuum is a dendrite if it is hereditarily decomposable, hereditarily unicoherent, arc-connected, and locally connected.

5. A continuum is a dendroid if it is hereditarily decomposable, hereditarily unicoherent, and arc-connected.

6. A map is simple if the inverse of each point in the range contains at most two points.

\section{References}

[1] K. Borsuk, A theorem on fixed points, Bull. Acad. Polon. Sci. Cl. III 2 (1954), 17-20.

[2] W. Dębski, J. Heath and J. Mioduszewski, Exactly two-to-one maps from continua onto some tree-like continua, Fund. Math. 141 (1991), 269-276.

[3] W. H. Gottschalk, On k-to-1 transformations, Bull. Amer. Math. Soc. 53 (1947), $168-169$.

[4] O. G. Harrold Jr., Exactly $(k, 1)$ transformations on connected linear graphs, Amer. J. Math. 62 (1940), 823-834.

[5] J. Heath, 2-to-1 maps with hereditarily indecomposable images, Proc. Amer. Math. Soc. 113 (1991), 839-846.

[6] J. Heath and V. Nall, Tree-like continua and 2-to-1 maps, ibid., to appear.

[7] W. T. Ingram, An atriodic tree-like continuum with positive span, Fund. Math. 77 (1972), 99-107.

[8] P. Minc, Bottlenecks in dendroids, Topology Appl. 129 (2003), 187-209.

[9] S. B. Nadler, Jr. and L. E. Ward, Jr., Concerning exactly $(n, 1)$ images of continua, Proc. Amer. Math. Soc. 87 (1983), 351-354. 
[10] R. E. Smithson, On the existence of exactly (2,1) maps, ibid. 99 (1987), 577-580.

Mathematics Department

Parker Hall

Auburn University

Auburn, AL 36849-5310, U.S.A.

E-mail: heathjw@auburn.edu
Department of Mathematics and Computer Science

University of Richmond Richmond, VA 23173, U.S.A.

E-mail:vnall@richmond.edu

Received 11 February 2003;

in revised form 6 November 2003 